\title{
Characterization of Breast Masses in Mammography using First Order Statistic
}

\author{
Umaima S. E. Ali ${ }^{1 *}$, Eman M. Algorashi ${ }^{1}$, Mohamed E. M. Gar-Elnabi ${ }^{1,2}$
}

${ }^{1}$ College of Medical Radiologic Science, Sudan University of Science and Technology, Khartoum, Sudan

${ }^{2}$ National university-Sudan, Faculty of Radiography \& medical imaging, Khartoum, Sudan

DOI: $10.36347 /$ sjams.2020.v08i10.004

| Received: 22.09.2020 | Accepted: 29.09.2020 | Published: 06.10.2020

*Corresponding author: Umaima Saad Elamin Ali

Abstract

Original Research Article

This study concern to characterize the breast masses in mammography were defining the breast tissues to tumor, gland, fat and connective tissue, at cancer diagnostic medical center and carried out using Interactive Data Language [IDL] program as platform for the generated codes. The texture analysis technique used to find the gray level variation in mammography images. Analyzing the image with Interactive Data Language IDL software to measure the grey level variation of mammography images. The result of the classification showed that the breast areas were classified well from the rest of the tissues although it has characteristics mostly similar to surrounding tissue. Several texture features are introduced from first order statistics and the classification score matrix generated by linear discriminate analysis and the classification accuracy of breast tissues classified to Tumor $96.8 \%$, gland $57.9 \%$, fat 98.9 , While the connective tissue showed a classification accuracy $98.5 \%$. The overall classification accuracy of breast area $94.0 \%$. These relationships are stored in a Texture Dictionary that can be later used to automatically annotate new mammography images with the appropriate breast area names.

Keywords: Breast mass, connective tissue, breast fatty, FNAC.

Copyright @ 2020: This is an open-access article distributed under the terms of the Creative Commons Attribution license which permits unrestricted use, distribution, and reproduction in any medium for non-commercial use (NonCommercial, or CC-BY-NC) provided the original author and source are credited.

\section{INTRODUCTION}

Mammography is the most effective modality to early detect of breast cancer [1] However, the positive predictive value of mammographic diagnosis is only about $15 \%-30 \%$ [2]. As the number of patients undergoing mammography increases, it will be increasingly important to improve the positive predictive value of this procedure in order to decrease patient discomfort and costs [3]. Recent studies have shown that mammography is sensitive in diagnosis and screening of breast cancer, but with a high falsepositive rate [4]. According to the traumatic nature and high cost of histopathology, can be results in develop computer-based methods to accurately differentiate the benign masses from malignant tumors. this computerized algorithm can assist radiologists in classification of mammographic abnormalities that can decrease benign biopsies [3], all this can be helpful in the primary or secondary screening of mammograms, and provide objective tools to assist radiologists in analyzing difficult cases and in deciding on biopsy recommendations. It is known that a small but significant number of cancer cases detected in screening programs have prompts visible in earlier screening examinations[5].
Most of the reported attempt to identify the concepts to distinguish between benign and malignant masses. Moreover, mammographic mass boundaries are usually blurry, making it difficult for automated detection schemes to accurately detect the mass boundaries. For these reasons, features quantifying the gradient and textural information contained by masses are needed to perform classification independent of shape[6].

Benign masses are more homogeneous as compared to malignant tumors in terms of density distribution[6]. Most benign masses have well-defined and clear boundaries, in contrast to irregular or illdefined boundaries of malignant tumors. However, some benign masses like fibroadenomas and cystic masses may also have ill-defined boundaries [7].

\section{Irst-Order Statistical Texture Analysis}

First-order texture analysis measures use the image histogram, or pixel occurrence probability, to calculate texture. The main advantage of this approach is its simplicity through the use of standard descriptors [e.g. mean and variance] to characterize the data [8]. 
However, the power of the approach for discriminating between unique textures is limited in certain applications because the method does not consider the spatial relationship, and correlation, between pixels. For any surface, or image, grey-levels are in the range $0 \leq \mathrm{i} \leq \mathrm{Ng}-1$, where $\mathrm{Ng}$ is the total number of distinct grey-levels. If $\mathrm{N}$ [i] is the number of pixels with intensity $\mathrm{i}$ and $\mathrm{M}$ is the total number of pixels in an image, it follows that the histogram, or pixel occurrence probability, is given by,

$$
P(i)-\frac{N(i)}{M}
$$

In general, seven features commonly used to describe the properties of the image histogram, and therefore image texture, are computed. These are: mean; variance; coarseness; skewness; kurtosis; energy; and entropy. These textural features include First order statistics; [coefficient of variation, stander deviation, variance, signal, energy, and entropy].

\section{Examination technique and protocol used}

The patient should remove any clothing, jewelry, or other objects that might interfere with the procedure, and remove any clothing from the patient waist up and ask the patient if they have noticed any lumps or other changes in either breast. If so, an adhesive marker will be placed on the spot (s) prior to the procedure.

The patient will stand in front of a mammography machine and the breast will be placed on the X-ray plate. In order to position the breast for optimal imaging, the technologist may examine and/or palpate the breast before placing it on the plate. An adhesive marker may be applied to any moles, scars, or other spots that might interfere with the breast image. A separate flat plate, often made of plastic, will be brought down on top of the breast to compress it gently against the X-ray plate. Compression of the breast is required in order to minimize the amount of radiation used and to ensure optimal visualization of the breast tissue. You may feel some discomfort during this time and asked the patient to hold a breath while the image is being taken, the radiologic technologist will step behind a protective window while the image is taken. Two pictures at different angles will be taken of each breast, requiring the breasts to be repositioned between pictures.

\section{Methodology}

This study carried out to evaluate the role of texture analysis technique in detection of and outlining the breast masses related to its textural feature. The sample is 155 patients and the data collected randomly from X-ray department at cancer diagnostic medical center. The patient under examination must perform $\mathrm{x}$-ray mammography, and FNAC of breast mass in order to identify the tumor feature and type, determine tumor site and size then the patient image will analyzed by using of IDL program for textural analysis.

Once we need faster and accurate diagnostic modalities in this situation in order to have high diagnostic accuracy in assessing breast tumors and therefore using this scan to plan patient for treatment which need Avery accurate delineation of tumor edges in case of CTV and planning target volume in order to deliver sufficient dose in case of radiation to the both volumes and increase therapeutic and diagnostic radio.

\section{RESULTS AND DisCUSSION}

Table-1: A confusion matrix shows the classification accuracy of the original classes versus the predicted membership according to linear discriminant functions [multiple linear regression equation]

\begin{tabular}{|c|c|c|c|c|c|c|}
\hline \multicolumn{2}{|c|}{ Classes } & \multicolumn{4}{|c|}{ Predicted Group Membership } & Total \\
\hline \multirow{4}{*}{$\%$} & Original Group & Tumor & Gland & Fat & Connective T & \\
\cline { 2 - 7 } & Tumor & $\underline{96.8}$ & .0 & .0 & 3.2 & 100.0 \\
\cline { 2 - 7 } & Gland & 42.1 & $\underline{57.9}$ & .0 & .0 & 100.0 \\
\cline { 2 - 7 } & Fat & .0 & .0 & $\underline{98.9}$ & 1.1 & 100.0 \\
\cline { 2 - 7 } & \multirow{2}{*}{ Connective T } & .0 & .0 & 1.5 & $\underline{98.5}$ & 100.0 \\
\hline
\end{tabular}

$94.0 \%$ of original grouped cases correctly classified

Table [1] show classification score matrix generated by linear discriminate analysis and the overall classification accuracy of breast masses $94.0 \%$, were the classification accuracy of gland $86.8 \%$, fat $98.2 \%$, connective tissue $98.9 \%$, While the tumor showed a classification accuracy $98.5 \%$. 


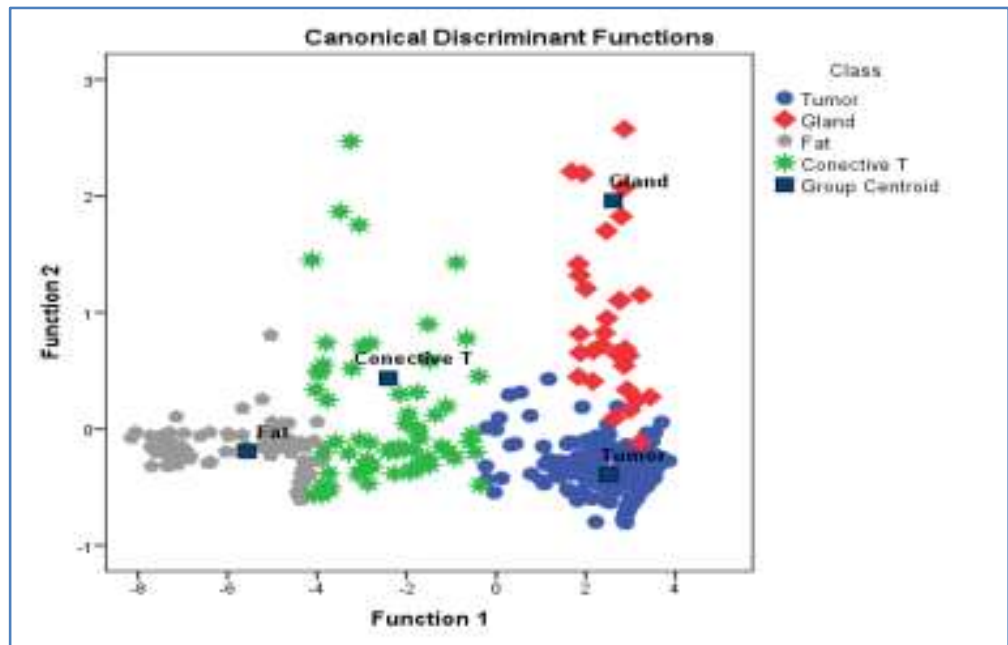

Fig-1: Scatter plot demonstrates the distribution of four Classes according to their textural feature using linear discriminate analysis functions

The classification showed that the breast although it has characteristics mostly similar to tissues were classified well from the rest of the tissues surrounding tissue.

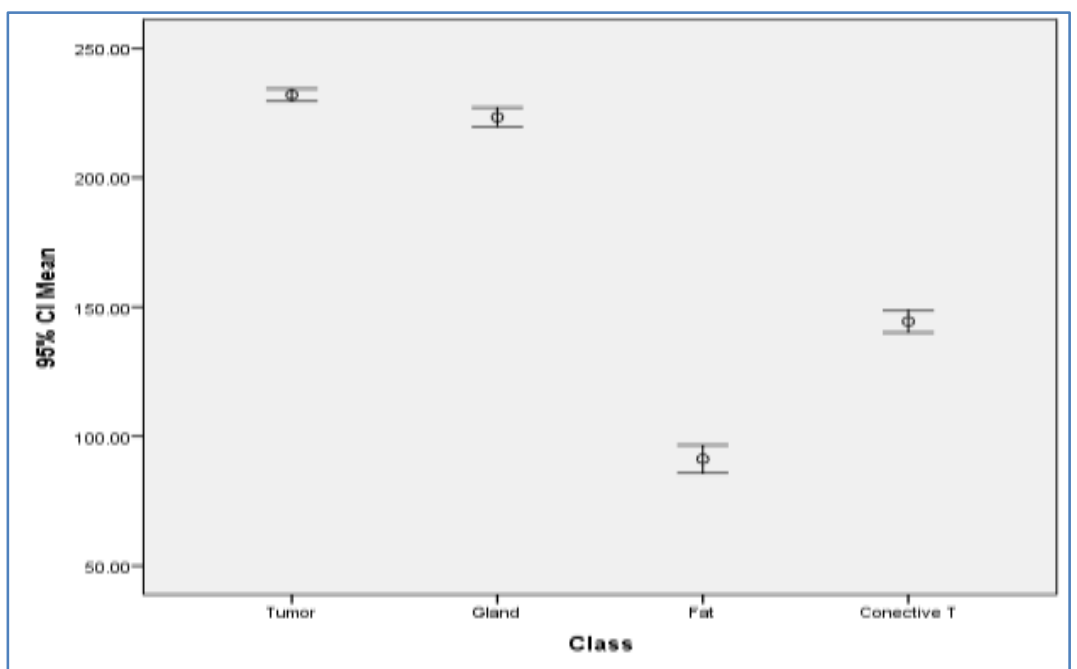

Fig-2: Show error bar plot for the CI mean textural features that selected by the linear stepwise discriminate function as a discriminate between all features. From the discriminate power point of view in respect to the applied features the mean can differentiate between all the classes successfully

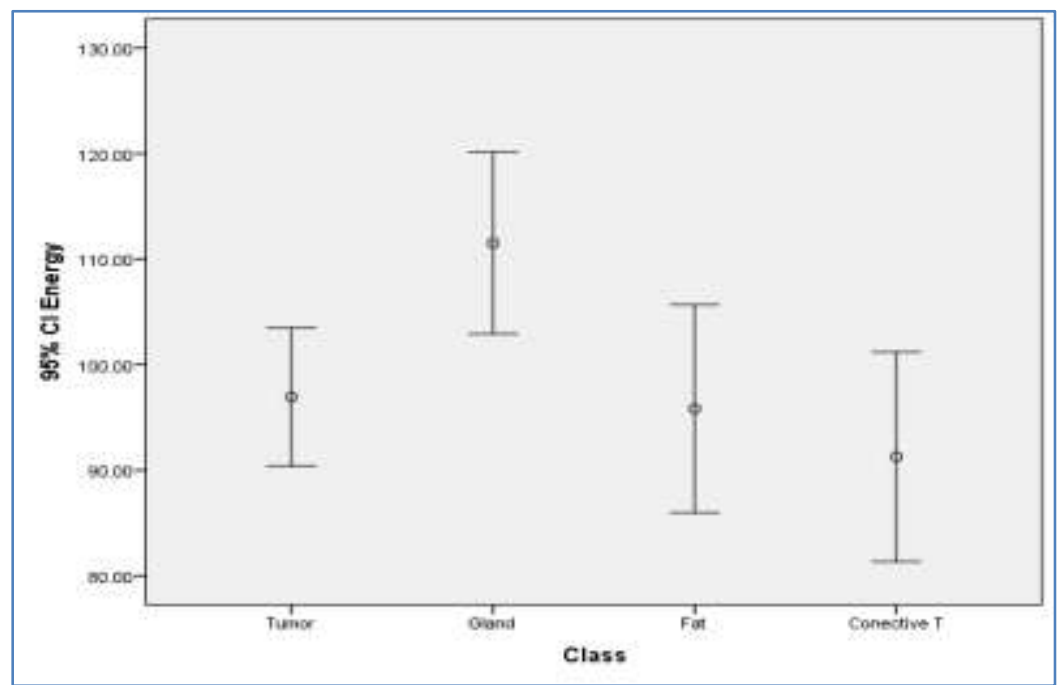

Fig-3: Show error bar plot for the CI energy textural features that selected by the linear stepwise discriminate function as a discriminate feature where it discriminates between all features 


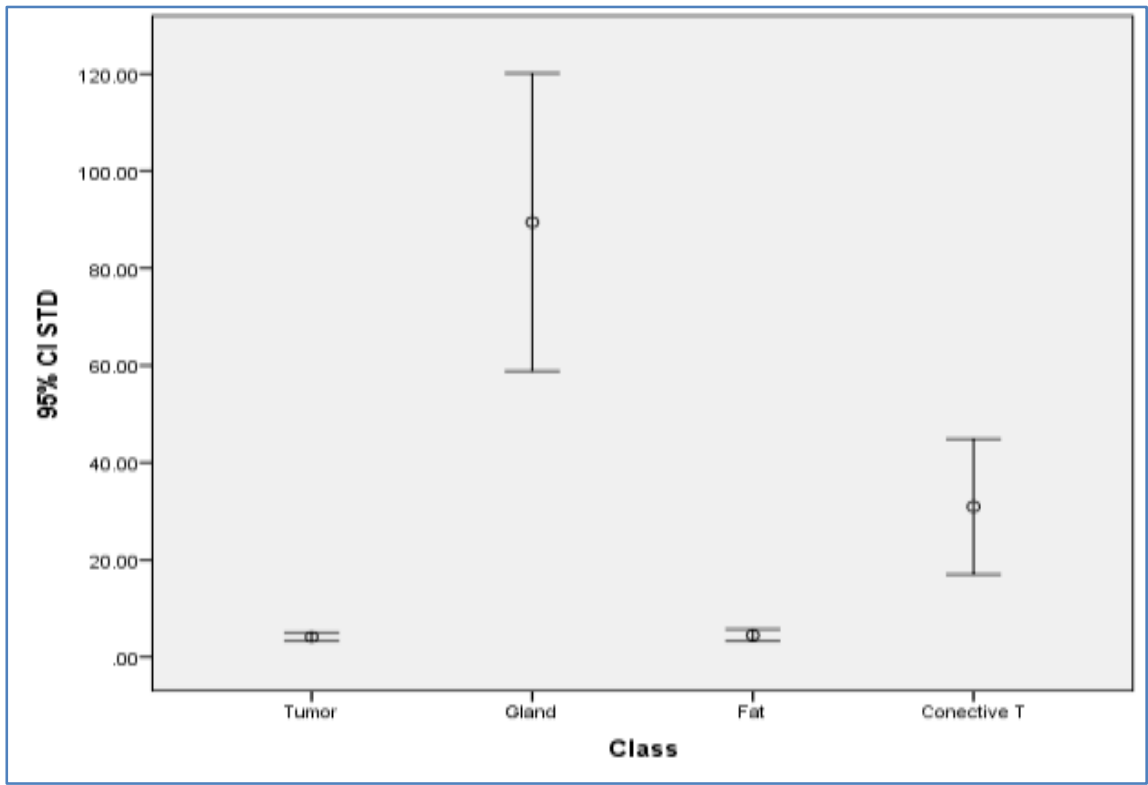

Fig-4: show error bar plot for the CI standard deviation textural features that selected by the linear stepwise discriminate function to discriminate between all features. From the discriminate power point of view in respect to the applied features the STD can differentiate between all the classes successfully

Comparable with other studies; Mona E. Elbashier 2017[9], discussed the Characterization of Pancreas at Diabetic Patients in CT Images using Texture Analysis with Gray Level Run Length Matrix. The results showed a good classification were the pancreas head $89.2 \%$, body 93.6 and the tail classification accuracy $93.5 \%$. The overall classification accuracy of pancreas area $92.0 \%$. And Simaa Hamid 2016 [10] studied the Characterization of Multiple Sclerosis Lesion in MR Imaging Using Texture Analysis, with GLRLM and its features seem very useful in texture classification, and the classification accuracy of multi sclerosis $96.9 \%$, white matter 93.8 $\%$, grey matter $92.6 \%$ and the CSF $100 \%$, with overall accuracy classification 95.2\%. Amna M. Ahmed 2017 [11] discussed the Characterization of Corpus Collosum in MR Images using Texture Analysis, the first order statistic and features give classification accuracy of corpus collosum parts for splenium $100.0 \%$, trunk $76.5 \%$ and the genu classification accuracy $97.0 \%$. The overall classification accuracy of corpus collosum area $96.2 \%$.

\section{Conclusion}

The classification processes of breast masses in mammography were defining the breast tissues to tumor, gland, fat and connective tissue and carried out using Interactive Data Language [IDL] program as platform for the generated codes. The result of the classification showed that the breast areas were classified well from the rest of the tissues although it has characteristics mostly similar to surrounding tissue.

Several texture features are introduced from first order statistics and the classification score matrix generated by linear discriminate analysis and the overall classification accuracy of breast tissues classified to Tumor $96.8 \%$, gland $57.9 \%$, fat 98.9 , While the connective tissue showed a classification accuracy $98.5 \%$. The overall classification accuracy of breast area $94.0 \%$.

Using linear discrimination analysis generated a classification function which can be used to classify other image into the mention classes as using the following multi regression equation;

\footnotetext{
Tumor $=[$ mean $\times 0.812]+[S T D \times 0.073]+[$ energy $\times 0.177]-104.285$

Gland $\quad=[$ mean $\times 0.815]+[S T D \times 0.137]+[$ energy $\times 0.186]-108.853$

Fat $\quad=[$ mean $\times 0.351]+[S T D \times 0.035]+[$ energy $\times 0.102]-22.353$

Connective $T=[$ mean $\times 0.529]+[S T D \times 0.069]+[$ energy $\times 0.129]-46.583$
}

\section{REFERENCES}

1. Ariel IM, Clearly J. Breast cancer: diagnosis and treatment; 1987.

2. Kopans DB. The positive predictive value of mammography. AJR American journal of roentgenology. 1992;158(3):521-6.
3. Sahiner B, Chan HP, Petrick N, Helvie MA, Goodsitt MM. Computerized characterization of masses on mammograms: The rubber band straightening transform and texture analysis Medical Physics. 1998;25(4):516-26.

4. Sterns EE. Relation between clinical and mammographic diagnosis of breast problems and 
the cancer/biopsy rate. Canadian journal of surgery. 1996;39(2):128.

5. Burrell HC, Sibbering DM, Wilson AR, Pinder SE, Evans AJ, Yeoman LJ, Elston CW, Ellis IO, Blamey RW, Robertson JF. Screening interval breast cancers: mammographic features and prognosis factors. Radiology. 1996 Jun;199(3):8117.

6. Mudigonda NR, Rangayyan R, Desautels JL. Gradient and texture analysis for the classification of mammographic masses. IEEE transactions on medical imaging. 2000 Oct;19(10):1032-43.

7. Homer MJ. Mammographic interpretation: a practical approach: McGraw-Hill; 1997.

8. Press W, Flanner y B, Teulkolsky S and Vetterling W. Numerical recipes in $\mathrm{C}$ : the art of scientific computing. Cambridge Universit y Press, UK.

9. Mona E. Elbashier, SuhaibAlameen, Caroline Edward Ayad, Mohamed E. M. Gar-Elnabi, Characterization of Pancreas at Diabetic Patients in CT Images using Texture Analysis, International Journals of Advanced Research in Computer Science and Software Engineering. 2017; 7(7): 812.

10. Hamid S, Gar-Elnabi ME, Alameen S. Characterization of Multiple Sclerosis Lesionin MR Imaging Using Texture Analysis.

11. Amna M. Ahmed, Suhaib Alameen, Caroline Edward Ayad and Mohamed EM. Gar-Elnabi, characterization of corpus collosum in $\mathrm{mr}$ images using texture analysis, Asian Journal of Science and Technology. 2017; 08(10): 5871-5873. 